\title{
Glomerular-specific alterations of VEGF-A expression lead to distinct congenital and acquired renal diseases
}

\author{
Vera Eremina, ${ }^{1}$ Manish Sood,${ }^{1}$ Jody Haigh, ${ }^{1}$ András Nagy, ${ }^{1}$ Ginette Lajoie, ${ }^{2}$ \\ Napoleone Ferrara, ${ }^{3}$ Hans-Peter Gerber, ${ }^{3}$ Yamato Kikkawa, ${ }^{4}$ Jeffrey H. Miner, ${ }^{4}$ \\ and Susan E. Quaggin ${ }^{1,5}$
}

\begin{abstract}
${ }^{1}$ The Samuel Lunenfeld Research Institute, Mount Sinai Hospital, Toronto, Ontario, Canada ${ }^{2}$ Princess Margaret Hospital, University Health Network, Department of Pathology, Toronto, Ontario, Canada ${ }^{3}$ Department of Molecular Oncology, Genentech Inc., South San Francisco, California, USA

${ }^{4}$ Department of Medicine, Washington University School of Medicine, Renal Division, St. Louis, Missouri, USA

${ }^{5}$ St. Michael's Hospital, Toronto, Ontario, Canada
\end{abstract}

\begin{abstract}
Kidney disease affects over 20 million people in the United States alone. Although the causes of renal failure are diverse, the glomerular filtration barrier is often the target of injury. Dysregulation of VEGF expression within the glomerulus has been demonstrated in a wide range of primary and acquired renal diseases, although the significance of these changes is unknown. In the glomerulus, VEGF-A is highly expressed in podocytes that make up a major portion of the barrier between the blood and urinary spaces. In this paper, we show that glomerular-selective deletion or overexpression of VEGF-A leads to glomerular disease in mice. Podocyte-specific heterozygosity for VEGF-A resulted in renal disease by 2.5 weeks of age, characterized by proteinuria and endotheliosis, the renal lesion seen in preeclampsia. Homozygous deletion of VEGF-A in glomeruli resulted in perinatal lethality. Mutant kidneys failed to develop a filtration barrier due to defects in endothelial cell migration, differentiation, and survival. In contrast, podocyte-specific overexpression of the VEGF-164 isoform led to a striking collapsing glomerulopathy, the lesion seen in HIV-associated nephropathy. Our data demonstrate that tight regulation of VEGF-A signaling is critical for establishment and maintenance of the glomerular filtration barrier and strongly supports a pivotal role for VEGF-A in renal disease.

J. Clin. Invest. 111:707-716 (2003). doi:10.1172/JCI200317423.
\end{abstract}

\section{Introduction}

Glomeruli are highly specialized filtration barriers between the blood and urinary space. Each day, approximately $180 \mathrm{l}$ of blood passes through these filters in the average adult human kidney. Although water and small solutes must pass freely through this barrier, critical blood proteins such as albumin and blood clotting factors must not. The filter has a number of unique characteristics that provide the essential properties for this renal filtration process and include highly specialized glomerular visceral epithelial cells (podocytes), a fenestrated glomerular capillary endothelial system, and intervening glomerular basement membrane (GBM) that is produced by both the podocytes and the

Received for publication November 18, 2002, and accepted in revised form January 15, 2003.

Address correspondence to: Susan E. Quaggin, The Samuel Lunenfeld Research Institute, Room 871Q, Mount Sinai Hospital, 600 University Avenue, Toronto, Ontario M5G 1X5, Canada. Phone: (416) 586-4800 ext. 2859; Fax: (416) 586-8588; E-mail: quaggin@mshri.on.ca.

Conflict of interest: The authors have declared that no conflict of interest exists.

Nonstandard abbreviations used: glomerular basement membrane (GBM); fetal liver kinase 1 (Flk1); fms-like tyrosine kinase 1 (Flt1); HIV-associated nephropathy (HIVAN); Wilms tumor suppressor gene (WT1); $\alpha$-smooth muscle actin (VSMA). endothelial cells $(1,2)$ (Figure 1a). This filtration barrier is the target of injury and ultimate scarring in a wide variety of kidney diseases (3-8).

During glomerular development, the podocytes express numerous vascular growth factors such as VEGF-A, while the glomerular endothelial cells express the VEGF receptors fetal liver kinase 1 (Flk1) and fmslike tyrosine kinase 1 (Flt1) (9). In addition, the podocytes are geographically situated at the developing vascular cleft adjacent to incoming endothelial cells $(2,10)$ (Figure 1b). The location and gene expression profile of podocytes suggests that they are required to provide migratory cues to glomerular endothelial cells to establish the renal filtration barrier. Furthermore, similar to other fenestrated vascular beds in the body, podocytes continue to express VEGF-A in the mature glomerulus. This suggests that VEGF plays a role in maintaining the filtration barrier either through survival, proliferation, and/or differentiation cues to the adjacent specialized endothelia.

It is clear that VEGF is a critical mediator of vasculogenesis as heterozygous and homozygous null VEGF-A mice die with major vascular defects at 11.5 and 9.5 days after coitus, respectively $(11,12)$. However, its later role in specific vascular beds, such as the glomerulus, is less clear. Although dysregulation of VEGF-A expression 
has been demonstrated in a number of renal diseases, the significance of these changes is presently unknown.

To determine the role of VEGF-A in the glomerular filtration barrier, we generated mice with gain or loss of function of VEGF specifically in the podocyte, thus avoiding the embryo-lethal effects. The distinct glomerular-specific haploinsufficient and null phenotypes observed in this study demonstrate for the first time that the "dose" of VEGF is critical in the establishment and maintenance of later vascular beds, as it is in vascular formation during earlier stages of embryogenesis.

By 2.5 weeks of age, mice with podocyte-specific heterozygosity for VEGF developed endotheliosis and "bloodless glomeruli," the renal lesion seen in preeclampsia, which progressed to nephrotic syndrome, a common glomerular syndrome seen in humans (13), and end-stage kidney failure by 9-12 weeks. The podocyte-specific homozygotes died at birth or within 18 hours of birth with hydrops (generalized swelling), kidney failure, and grossly abnormal glomeruli that lack mature endothelial cells. In contrast, overexpression of the 164 isoform of VEGF-A in podocytes also led to end-stage renal failure due to a collapsing glomerulopathy, which is the pathologic lesion seen in HIV-associated nephropathy (HIVAN) (14). This demonstrates that there is an ongoing requirement for tight regulation of VEGF signaling between the podocyte and glomerular endothelium; disruption in this regulation leads to dramatic and distinct renal phenotypes that are determined by the glomerular VEGF dose and suggests that VEGF is pivotal in a wide variety of renal diseases.

\section{Methods}

Cell-specific gene targeting. Three independent podocytespecific Cre recombinase murine lines, A15, GG8, and V9, which all demonstrated $100 \%$ excision when crossed to the Z/EG reporter mouse strain (15), were bred to the floxed VEGF-A mouse (Figure 1c). The VEGF-A mouse has loxP sites inserted around the third exon (16). Site-specific recombination between the loxP sites of the VEGF gene results in a null VEGF allele (16).

To generate homozygous floxed VEGF-Cre recombinase mice, bitransgenic mice carrying both a nephrinCre transgene and one floxed VEGF-A allele were bred to homozygous floxed VEGF-A mice.

To generate transgenic founders that overexpress the 164 isoform of VEGF-A, a 645-bp fragment of the VEGF gene, including a Kozak consensus sequence (nucleotides 78-669 of GenBank accession no. NM009505) and initiating ATG, were subcloned into the XhoI and XbaI sites of a PNXRS vector between a $4.125-\mathrm{kb} 5^{\prime}$ fragment of the murine nephrin gene that is capable of podocyte-specific expression in vivo in the kidney (17) and a $0.97-\mathrm{kb}$ poly(A) signal from the SV40 polyoma virus (Figure 1e).

Genotyping. Genomic DNA was isolated from mouse tails as described. The nephrin-Cre transgenic mice were generated and genotyped as previously described (17). Floxed VEGF mice were received from Napoleone Ferrara (Genentech Inc.). Presence of the floxed VEGF gene was detected by PCR using the oligonucleotide primers muVEGF 419.F (5'-CCTGGCCCTCAAGTACACCTT-3') and muVEGF 567.R (5'-TCCGTACGACGCATTTCTAG-3') (both from Sigma-Genosys, The Woodlands, Texas, USA), which generate a 148-bp fragment of the VEGF allele in the presence of the loxP-1 site and a DNA fragment that is approximately $40 \mathrm{bp}$ shorter than for the wild-type allele (16).

To identify founder mouse lines that carried the nephrin-VEGF-164 transgene, Southern blot analysis was performed. Briefly, the DNA was digested with EcoRI; the probe used was the 645-bp fragment encoding the VEGF-164 cDNA that recognized a $1.3-\mathrm{kb}$ genomic fragment in the transgenic founders. To estimate transgene copy number, $1 \mu \mathrm{g}, 2 \mu \mathrm{g}$, and $5 \mu \mathrm{g}$ of genomic DNA from the transgenic founder or wild-type mice was blotted on Biodyne B membrane (P/N 60207, Pall Gelman Laboratory, Ann Arbor, Michigan, USA) and hybridized with the VEGF cDNA probe described above. The signal was quantified using the Quantity One quantitation software program (4.2.1 version) (BioRad Laboratories, Hercules, California, USA) according to the manufacturer's instructions.

Phenotypic analysis. Urine was collected passively in an Eppendorf tube from 0-, 3-, 6-, and 9-week-old mice. A urine dipstick (Chemstrip 5L; Roche Diagnostics Corp., Indianapolis, Indiana, USA) was used to detect the presence or absence of protein and red blood cells in the urine. The standard colorimetric assay was performed according to the manufacturer's instructions. In addition, $2 \mu \mathrm{l}$ of urine from transgenic or control mice was placed in $18 \mu \mathrm{l}$ of Laemmli buffer (18), boiled, and loaded on a 12\% SDS-PAGE gel. An SDS-PAGE low-range protein standard (BioRad Laboratories Inc., Hercules, California, USA) was loaded in the first lane of the gel.

Blood samples were taken with a heparinized capillary tube by femoral vein stab after warming. A total of $120 \mu \mathrm{l}$ of blood was collected and creatinine, urea, and blood chemistry measurements were recorded using a Stat Profile M7 (Nova Biomedical Corp., Waltham, Massachusetts, USA). The CBC (total blood count) was performed on a Coulter Counter (AcT diff; Beckman Coulter Canada, Ontario, Canada).

Histologic analysis. Embryonic tissues for histologic analysis were dissected, fixed in 10\% formalin/PBS, and embedded in paraffin. Sections $4 \mu \mathrm{m}$ thick were cut. Sections were stained with H\&E, examined, and photographed with a DC200 Leica camera and Leica DMLB microscope (Leica Microsystems Inc., Deerfield, Illinois, USA). Tissue for electron microscopy was fixed in $1.5 \%$ glutaraldehyde, embedded in Spurr (Canemco Inc., Saint-Laurent, Quebec, Canada), and sectioned.

In situ hybridization and immunohistochemistry. Kidneys were dissected from mice on postnatal day 0 and at 1 week, 3 weeks, 6 weeks, or 9 weeks of age. Kidneys were 
washed briefly in RNase-free PBS and fixed overnight in DEPC-treated 4\% paraformaldehyde. These tissues were then placed in 30\% sucrose for 12-24 hours, embedded in Tissue-Tek OCT 4583 compound (Sakura Finetek USA Inc., Torrance, California, USA) and snap frozen. Ten-micron tissue samples were cut on a Leica Jung cryostat (model CM3050; Leica Microsystems Inc.) and transferred to Superfrost microscope slides (Fisher Scientific Co., Pittsburgh, Pennsylvania, USA). The slides were stored at $-20^{\circ} \mathrm{C}$ until needed. Digoxigenin-labeled probes were prepared according to the Roche Molecular Biochemicals protocol (Roche Molecular Biochemicals, Mannheim, Germany). Probes used for in situ analysis were nephrin (19), Wilms tumor suppressor gene (WT1; a kind gift of J. Kreidberg, Children's Hospital, Boston, Massachusetts, USA), podocin (a kind gift from C. Antignac, Institut National de la Santé et de la Recherche Médicale, Paris, France), $\alpha$-smooth muscle actin (VSMA; a kind gift from P. Igarashi, Southwestern University, Dallas, Texas, USA), VEGF-A (kind gift of A. Nagy, Samuel Lunenfeld Research Institute, Toronto, Canada). Details of the in situ analysis protocol may be obtained upon request.

Immunostaining was performed with antibodies to WT1 and PECAM as described (20).

\section{Results}

Mice that are heterozygous for VEGF-A in the podocyte develop endotheliosis and nephrotic syndrome. To determine whether there is any phenotype resulting from a reduction in VEGF-A gene dose within the podocyte, we used the Cre-loxP system. Nephrin-Cre recombinase mice were generated in our laboratory and are capable of site-specific recombination in $100 \%$ of podocytes at the capillary-loop stage in vivo (17). We generated mice that were heterozygous for the floxed VEGF-A allele and carried the nephrin-Cre transgene VEGF-loxP $P^{+/}, \mathrm{Neph}-\mathrm{Cre}^{+/-}$ a
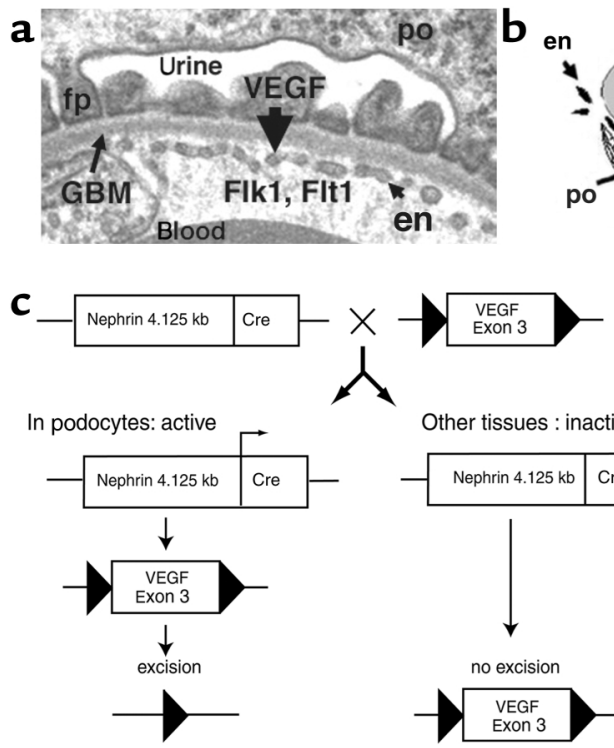

e

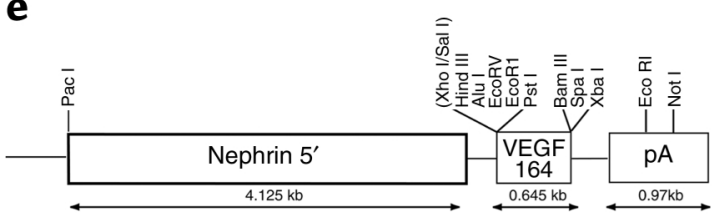

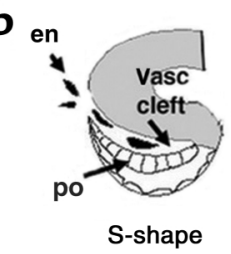

Other tissues : inactive
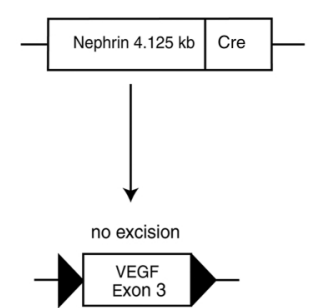

$\stackrel{0.645 \mathrm{~kb}}{\longrightarrow}$

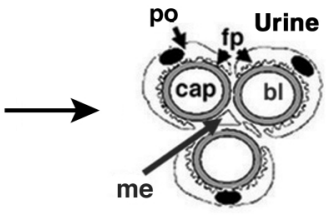

Mature glomerulus
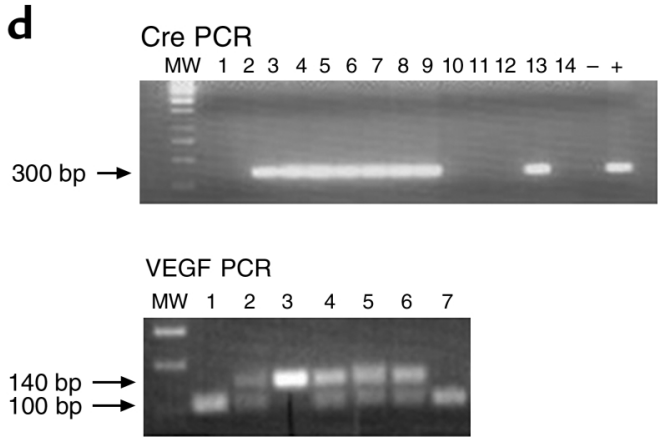

f

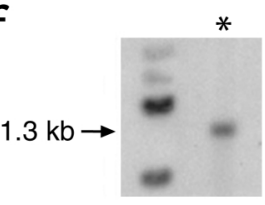

g

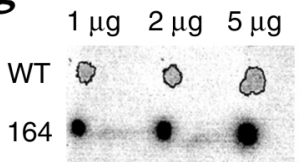

\section{Figure 1}

Expression and genomic targeting of VEGF-A within the glomerular filtration barrier. (a) Transmission electron micrograph of the glomerular filtration barrier that consists of podocytes (po) and their specialized foot processes ( $\mathrm{fp}$ ), fenestrated endothelium (en), and intervening GBM. VEGF-A is produced in the podocyte; the VEGF receptors Flk1 and Flt1 are expressed in the adjacent endothelial cells. (b) Development of the glomerular filtration barrier. In the S-shape stage, podocyte precursors (po) express VEGF-A. Endothelial cells (en) that express the VEGF receptors migrate into the vascular (Vasc) cleft and differentiate in direct apposition to podocytes. In the mature glomerulus, the fenestrated endothelial capillary loops (cap) remain in intimate contact with the VEGF-expressing podocytes (po). Mesangial cells (me) provide support to the capillary tuft. Urine is formed as blood (bl) is filtered from the capillaries, across the GBM, and through slit diaphragms that connect adjacent podocyte foot processes (fp). (c) Scheme to generate heterozygous and homozygous podocyte-specific VEGF knockout mice. Triangles are 34 bp loxP sites. (d) The Cre recombinase transgene was identified as a 300 bp PCR product. The floxed VEGF allele measures 140 bp by PCR analysis, whereas the wild-type allele measures $100 \mathrm{bp}$. MW, molecular weight markers. (e) Transgenic construct used to overexpress the 164-isoform of VEGF. pA, poly(A). (f) Presence of the transgenic VEGF-164 gene was identified as a 1.3-kb band $\left({ }^{*}\right)$ by Southern blot analysis. (g) Dot blot analysis of transgene copy number. The transgenic founder mice (164) demonstrated a 30-fold increase in copy number compared with the wild type. 


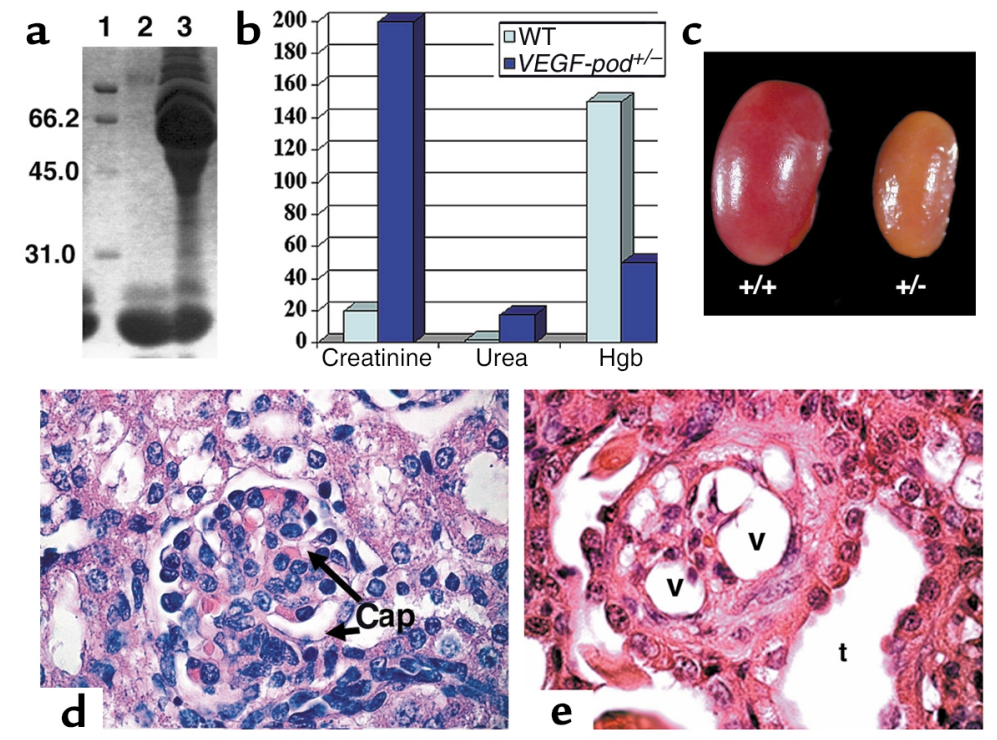

Figure 2

Heterozygous VEGF-lox $\mathrm{P}^{+/-}, \mathrm{Neph}$-Cre ${ }^{+/-}$mice develop nephrotic syndrome and endstage renal failure by 9 weeks of age. (a) SDS-PAGE analysis was performed using 2 $\mu \mathrm{l}$ of mouse urine. Lane 1 contains molecular weight markers, lane 2 shows urine from a VEGF-lox $\mathrm{P}^{-/-}, \mathrm{Neph}-\mathrm{Cre}^{+/-}$control aged 9 weeks, and lane 3 shows urine from a 9-week-old sick VEGF-lox $\mathrm{P}^{+/-}, \mathrm{Neph}-\mathrm{Cre}^{+/-}$animal. The presence of a large amount of albumin measuring $66.2 \mathrm{kDa}$ is identified in the sick mouse and demonstrates damage to the kidney filter. In contrast, low-molecular-weight proteins, which are normally found in mouse urine, are not different. (b) Bar graph showing elevated creatinine (more than ten times higher than normal), elevated urea, and decreased hemoglobin ( $\mathrm{Hgb})$ in VEGF-lox $\mathrm{P}^{+-}, \mathrm{Neph}-\mathrm{Cre}^{+/-}$mice (VEGF-pod ${ }^{+/-}$) at 9 weeks of age compared with VEGF-lox $\mathrm{P}^{+/-}, \mathrm{Neph}-\mathrm{Cre}^{-/-}$and VEGF-loxP-/-,Neph-Cre ${ }^{+/-}$mice (combined for analysis and considered as wild type). (c) Whole-mount image of a kidney from a sick 9-week-old VEGF-lox $\mathrm{P}^{+-}, \mathrm{Neph}-\mathrm{Cre}^{+/-}(+/-)$mouse compared with that of a wild-type littermate $(+/+)$. The affected kidney is pale and shrunken. Magnification: $\times 60$. (d) A wild-type glomerulus. Note the open capillary loops (Cap). $\times 350$. (e) A glomerulus from a heterozygous VEGF-A mouse. All the glomeruli are grossly distorted morphologically. Note the empty cytoplasmic vacuoles $(v)$ that are present in podocytes. No patent capillary loops can be seen. Dilated tubules $(t)$ can be seen and in most places are packed with proteinaceous material, consistent with nephrotic syndrome. Magnification: $\times 375$.

(Figure 1, c and d). They were born in the expected mendelian frequency but developed end-stage kidney failure by $9-12$ weeks of age.

Physical examination of mice at this stage showed that 30/30 of the VEGF-lox $\mathrm{P}^{+/-}, \mathrm{Neph}-\mathrm{Cre}^{+/-}$mice had lethargy and decreased skin turgor. Urinalysis was performed and showed $3.0 \mathrm{~g} / \mathrm{l}$ of protein (defined as "nephrotic range" proteinuria) and 250 red blood cells/ $\mu$ l of urine by dipstick analysis. SDS-PAGE analysis demonstrated massive albuminuria in all of these VEGF-lox $P^{+-}, \mathrm{Neph}-\mathrm{Cre}^{+/-}$mice (Figure 2a), which is pathognomonic for damage to the filtration barrier. Blood chemistry showed mice to have severely decreased renal function with an elevated serum creatinine that measured $200 \mu \mathrm{M}$, more than ten times the normal value, markedly elevated urea, and a normochromic, normocytic anemia consistent with end-stage kidney failure (Figure $2 \mathrm{~b}$ ). Of note, fragmentation of red blood cells was not observed on the blood smear. The mice did not demonstrate any gross signs of renal failure prior to 7-8 weeks of age.

At 9 weeks of age, the kidneys were pale and shrunken (Figure 2c). By light microscopy, the glomeruli looked histologically normal at birth and at 1, 3, and 6 weeks of age. However, by 9 weeks of age, the glomerular tufts were retracted with expansion of the mesangial matrix and were surrounded by podocytes containing large empty cytoplasmic vacuoles. The tubules were packed with protein (Figure $2 \mathrm{e}$ and data not shown).

Serial transmission EM studies demonstrated that the first detectable lesion occurred at 2.5 weeks of age with swelling of the endothelial cells (endotheliosis) and hyaline deposits (Figure 3a) that resemble the pathologic lesions seen in renal biopsies from patients with preeclampsia, a common disease of pregnancy (21). At this time, the podocytes and endothelial cells appeared ultrastructurally normal with well-formed foot processes and fenestrations, respectively. By 6.5 weeks of age, the GBM was expanded and endothelial fenestrations could no longer be identified (Figure 3b). By 9 weeks of age, the endothelial cells were necrotic and no podocyte foot processes could be identified (Figure $3 \mathrm{~b}$ ).

Molecular marker analysis confirmed the disappearance of differentiated podocytes with a complete absence of WT1, nephrin, and VEGF-A in the majority of glomeruli of terminally ill mice (Figure $4, \mathrm{~g}$ and $\mathrm{h}$, and data not shown). On occasion, a single cell could be identified that stained positively for these markers (Figure 4h). These markers were all present at birth and at 3 and 6 weeks of age (Figure 4, d-f and data not shown). The level of VEGF-A mRNA was consistently lower in the heterozygous VEGF glomeruli than in control glomeruli at the same developmental stage (Figure 4d). VSMA is not normally found in 9-week-old mesangial cells unless they are "activated" in glomerular injury; in the heterozygotes, occasional VSMA-positive cells were identified in the glomeruli (Figure 4i).

VEGF-A is required in the podocyte to establish the glomerular filtration barrier. In order to investigate the phenotype resulting from a complete absence of VEGF-A in the glomerulus, mice that were null for VEGF-A specifically in the podocyte (VEGF-lox $P^{+/+}, \mathrm{Neph}-\mathrm{Cre}^{+/-}$mice) were generated $(n=15)$. These mice were born at the expected mendelian frequency but died at birth or within 18 hours of birth. Some of these mice were born with hydrops that can be seen in infants with congenital nephrotic syndrome (22).

Light microscopy demonstrated that all of the null VEGF-A glomeruli were small with no or few distin- 
a
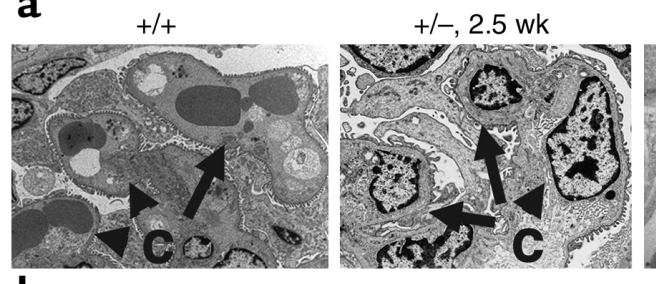

b

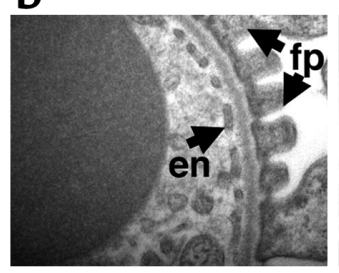

$+/+$

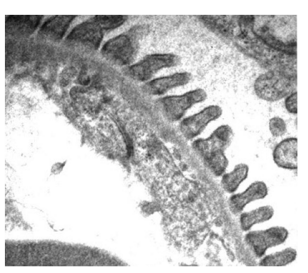

$+/-, 6.5 \mathrm{wk}$
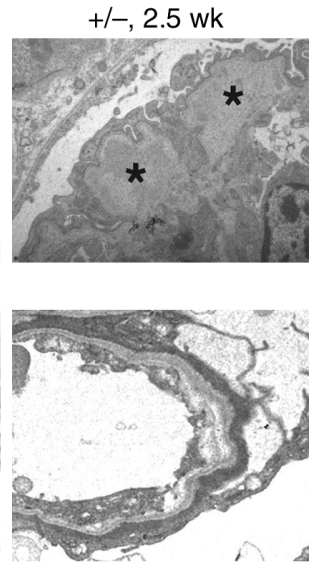

$+/-, 9$ wk

\section{Figure 3}

Heterozygous VEGF-lox ${ }^{+/-}, \mathrm{Neph}-\mathrm{Cre}^{+/-}$mice demonstrate endotheliosis and loss of fenestrations. (a) At 2.5 weeks of age, wild-type glomerular capillary loops (c) are open and contain numerous red blood cells. In contrast, podocytespecific VEGF-A heterozygotes (+/-) demonstrate bloodless glomeruli, and the capillary loops are filled with swollen endothelial cells, demonstrating endotheliosis, the classic renal lesion of preeclampsia. In addition, large subendothelial hyaline deposits $\left({ }^{*}\right)$ can be seen. (b) At 6.5 weeks of age, wild-type filtration barriers $(+/+)$ are characterized by fenestrated endothelial cells (en) and well-formed podocyte foot processes ( $\mathrm{fp}$ ). In the podocyte-specific heterozygotes $(+/-)$, the fenestrations are lost at 6.5 weeks of age, and by 9 weeks of age, the endothelial cells appear necrotic and no podocyte foot processes can be identified. guishable glomerular capillary loops. Additionally, podocytes were present but tended to pile up in several layers and lacked well-formed slit diaphragms, the specialized intercellular junctions found between foot processes (Figure 5a and data not shown).

Immunohistochemical analysis with an antibody to PECAM that recognizes a cell surface receptor on endothelial cells was performed. Although endothelial cells were present in most immature glomeruli, they were markedly reduced in number and mature glomeruli lacked endothelial cells altogether (Figure $5 b)$. BrdU labeling was performed; labeled endothelial cells were easily identified in the vascular clefts of wild-type S-shape stage glomeruli but were never observed in podocyte-specific VEGF-A null glomeruli (data not shown).

EM studies demonstrated widespread but not complete effacement of podocyte foot processes (not shown). Small capillary loops with a GBM could be identified in some glomeruli. However, this GBM failed

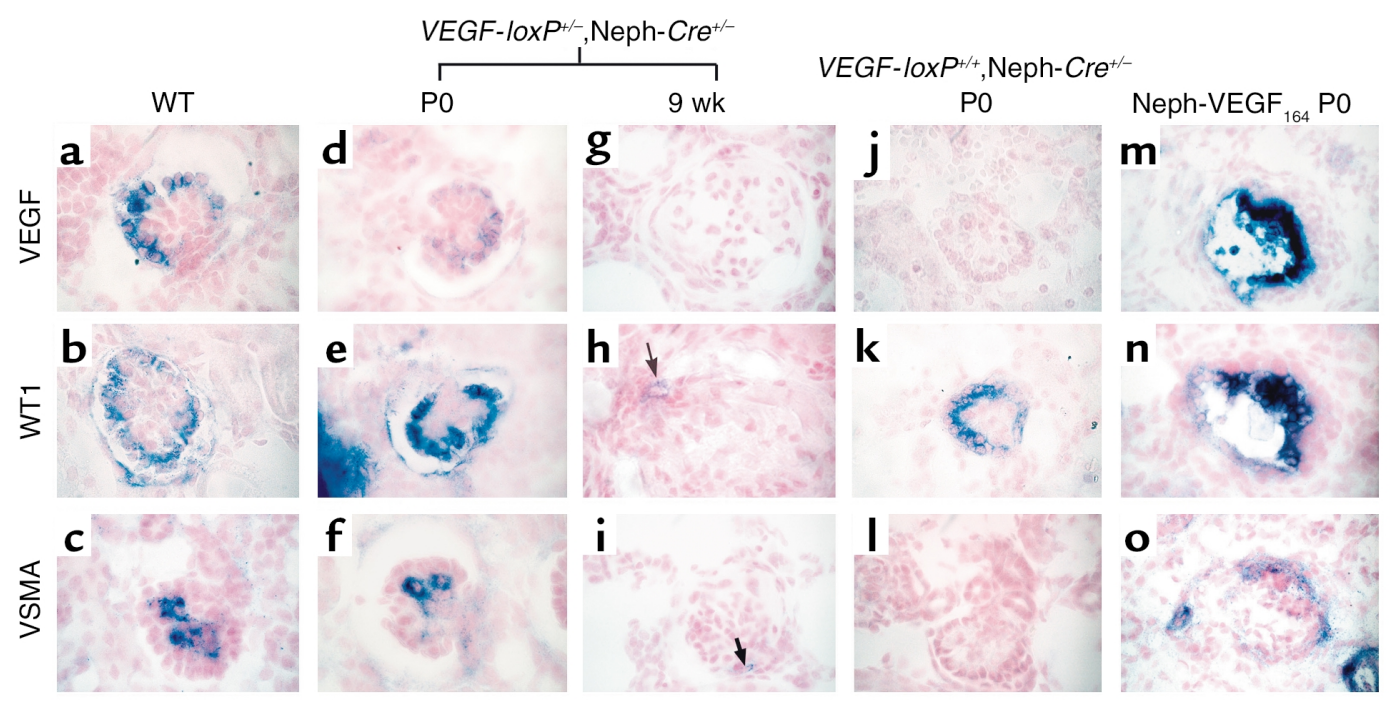

Figure 4

Digoxigenin-labeled in situ analysis of wild-type and mutant glomeruli. (a-c) Capillary loop-stage glomeruli from a newborn VEGF-loxP-/-, $\mathrm{Neph}-\mathrm{Cre}^{+/-}$control mouse demonstrate expression of VEGF-A and WT1 in podocytes, while VSMA is expressed in mesangial cells, which are found inside the glomerulus and are required to support the capillary structure. (d-f) At birth (P0), capillary loop-stage glomeruli from a heterozygous VEGF-lox $P^{+/-}, \mathrm{Neph}-\mathrm{Cre}^{+/-}$mouse demonstrate normal levels of expression of WT1 and VSMA, while VEGF expression is consistently reduced at the mRNA level compared with the wild-type controls. (g-i) By 9 weeks of age, the heterozygous VEGF mice are clinically unwell. At this time, most glomeruli demonstrate a complete absence of markers of podocyte differentiation (i.e., no VEGF or WT1; both are absent). In h, a single WT1-positive cell can be identified (arrow). (i) VSMA is not usually present in glomeruli at 9 weeks; however, occasional VSMA-pos-

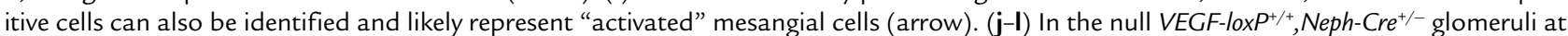
birth, no VEGF is seen in glomeruli as predicted due to podocyte-specific excision of both VEGF alleles. WT1 is present in differentiated podocytes. In contrast, VSMA is absent, demonstrating a defect in migration and/or differentiation of mesangial cells into the glomerulus. ( $\mathbf{m}-\mathbf{o}$ ) In the nephrin-VEGF-164 mouse, both VEGF and WT1 are expressed in podocytes present within collapsed glomeruli. VEGF is markedly upregulated. In addition, VSMA and mesangial cells are present but appear to surround the collapsed glomerulus in a crescent shape. Magnification: $\times 350$. 
a

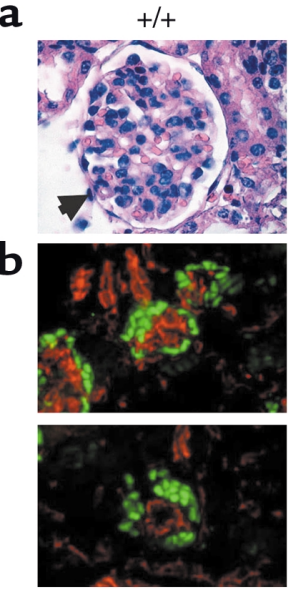

Immature

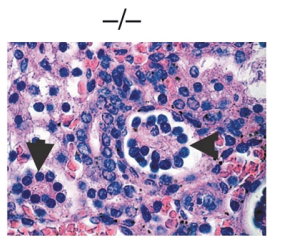

C $+/+$, immature
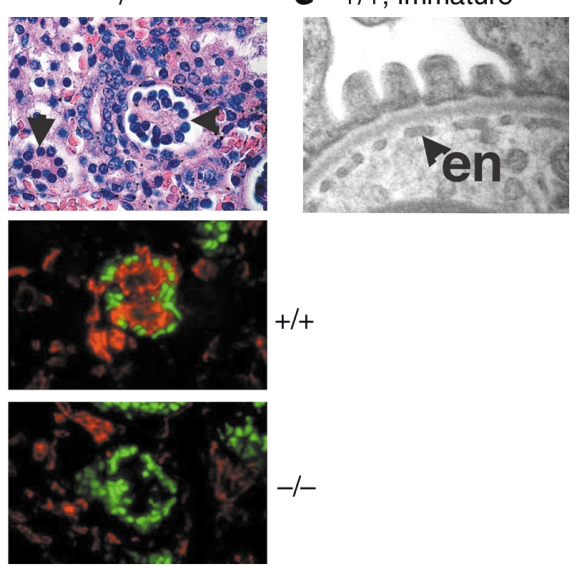

Mature
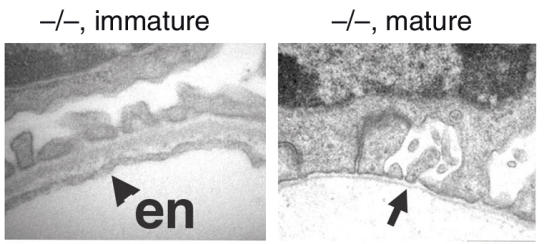

WT1/PECAM

\section{Figure 5}

VEGF-null glomeruli do not form filtration barriers or fenestrations within endothelial cells. (a) The wild-type $(+/+)$ glomerulus (arrow) has a lacy appearance due to open capillary loops. The VEGF-null glomeruli (-/-) fail to develop fully and lack visible capillary loops. Magnification: $\times 350$. (b) Immunohistochemical staining for WT1 (green), a marker for podocyte cells, and PECAM (red), a marker for endothelial cells, shows a reduced number of endothelial cells in immature (capillary loop-stage) VEGF-null glomeruli. In mature glomeruli, no endothelial cells remain. Magnification: $\times 300$. (c) Transmission EM of the filtration barrier in a wild-type $(+/+)$ glomerulus clearly demonstrates fenestrated endothelium at the late capillary-loop stage, whereas no fenestrations are observed in endothelial cells (en) found in corresponding late capillary loop-stage VEGF-null glomeruli. In mature VEGF-null glomeruli, the basement membrane is seen (arrow), but the endothelial cells are missing. Magnification: $\times 20,000$.

to fuse (data not shown). Endothelial cells were seen only rarely in capillary loop glomeruli and always lacked fenestrations (Figure 5c). In contrast, fenestrations were easily observed in endothelial cells of capillary loop-stage wild-type glomeruli (Figure 5c). Capillary loops in fully differentiated (mature) glomeruli demonstrated an absence of endothelial cells (Figure 5c).

In situ analysis showed that the podocytes that were present expressed markers of differentiation appropriately, including WT1 (Figure 4k), nephrin, and podocin (data not shown), although VEGF-A was absent due to genomic excision of the VEGF gene (Figure 4j). Of note, VSMA, a marker for mesangial cells, was absent from mutant glomeruli, although some desmin staining could be identified (Figure $4 \mathrm{l}$ and data not shown).

Upregulation of VEGF-A in podocytes leads to a collapsing glomerulopatby and death at 5 days of age. Given the distinct phenotypes observed when the dosage of VEGF is reduced by excising one or both alleles from the podocyte, we next sought to determine the effect of increasing the level of VEGF within the podocyte and its effect on the adjacent endothelium. Transgenic founder lines that overexpressed the 164 isoform of VEGF-A (nephrin-VEGF-164) under regulation of a $4.125-\mathrm{kb}$ podocyte-specific promoter from the murine nephrin gene (Figure 1e) were identified by Southern blot analysis (Figure 1f). Two independent founder mice were used for analysis. By dot blot analysis, each of these founder mice demonstrated a 30-fold increase in the VEGF copy number (Figure 1g).

The transgenic mice appeared normal at birth but became growth-retarded within 2 days. By 5 days of age, the mice were clinically unwell and demonstrated albuminuria by dipstick analysis.

Grossly, the kidneys appeared normal in size, were hyperemic, and demonstrated cortical hemorrhages (Figure 6, a and b). Light microscopy showed global collapse of the glomerular tuft and dilation of proximal tubules that were packed with protein (Figure 6d and data not shown). Complete collapse of the capillary loops was illustrated by silver methenamine staining that recognizes the GBM (Figure 6f).

The few visible patent capillary loops were larger in diameter (Figure 6h) and multiple endothelial cell nuclei were visible within them (Figure $6, \mathrm{~h}$ and $\mathrm{j}$ ) that were not seen in wild-type glomeruli (Figure 6, $g$ and i). Although multiple endothelial cell nuclei could be identified within the few remaining patent glomerular capillary loops by EM, virtually all of the loops were collapsed and no endothelial cells could be identified. In addition, podocytes were abnormal and could be seen detaching from the GBM (data not shown).

In situ analysis confirmed that the majority of cells within the collapsed tufts were podocytes that continued to express WT1 (Figure 4n) and nephrin (not shown) and very high levels of VEGF-A that were upregulated five- to tenfold (Figure $4 \mathrm{~m}$ and data not shown). Although mesangial cells were present as indicated by the presence of VSMA (Figure 4o), they were situated in a crescent shape at the periphery of the glomerulus.

\section{Discussion}

VEGF-A is a critical mediator of angiogenesis and vasculogenesis (11); both heterozygous and homozygous 
knockout mice die during embryogenesis due to major vascular defects. This demonstrates a dosage sensitivity for VEGF during development in the whole embryo (11). Other studies have shown that VEGF-A is required for the establishment and maintenance of endothelial fenestrae in vitro $(23,24)$.

Given the expression pattern of VEGF-A in developing and mature podocytes, which are located in direct apposition to fenestrated endothelial cells, and the fact that VEGF-A expression is associated with a variety of renal diseases, we hypothesized that VEGF-A is required in developing podocytes to establish and maintain the filtration barrier. To test this hypothesis, we used the Cre-loxP system to manipulate levels of VEGF-A expression specifically within the podocyte. The mice developed distinct haploinsufficient, null, and overexpression phenotypes. Thus, similar to vascular development in the early embryo, tight regulation of VEGF signaling is essential in the establishment of later vascular beds such as the glomerulus. In addition, sequential reduction in VEGF-A levels led to a loss of fenestrations or failure of fenestrations to form, definitively demonstrating for the first time a role for VEGF-A in maintenance of endothelial fenestrations in vivo. Finally, the distinct and dramatic renal phenotypes observed with each alteration of VEGF level suggest that VEGF signaling is pivotal in glomerular health and establish its role in the pathogenesis of glomerular disease.

Complete loss of VEGF-A in the glomerulus was $100 \%$ fatal in the perinatal period. The null VEGFlox $\mathrm{P}^{+/+}, \mathrm{Neph}-\mathrm{Cre}^{+/-}$mice died within 18 hours of birth with generalized swelling (hydrops) and a failure of the glomerular filtration barrier to form. Although occasional endothelial cells were identified in most but not

\section{Figure 6}

Mice that overexpress the 164 isoform of VEGF-A in their podocytes develop collapsing glomerulopathy. ( $\mathbf{a}$ and $\mathbf{b}$ ) Whole-mount images of VEGF-overexpressing kidneys at 5 days. The kidneys demonstrate many surface hemorrhages. (c) A glomerulus stained with $\mathrm{H} \& \mathrm{E}$ from a wild-type littermate. (d) A glomerulus from a transgenic VEGF-overexpressing mouse demonstrates global collapse of the capillary tuft toward the vascular pole of the glomerulus. A single patent capillary loop that appears dilated is identified (Cap). In addition, Bowman's space (BS) is enlarged. (e) A 5-day-old wild-type glomerulus is stained with silver methenamine that recognizes basement membranes (black). Note the intricate pattern of GBM that lines the capillary loops between endothelial cells and podocytes. (f) In contrast, a transgenic glomerulus demonstrates complete collapse of the capillary network. (g) A high-power view of the capillary loops (Cap) in a wild-type glomerulus. (h) In contrast, the few patent capillary loops identified at 5 days of age in the transgenic mice demonstrate increased diameter and multiple endothelial cell nuclei (arrowheads). (i) A wild-type capillary loop at 5 days of age. Note the fenestrated endothelium (arrow). Although a portion of an endothelial cell body is identified (arrowhead), glomerular endothelial cell nuclei are difficult to find on EM sections. (j) In a transgenic patent capillary loop at 5 days of age, three endothelial cell nuclei are easily identified (arrowheads). Magnification in $\mathbf{a}$ and $\mathbf{b}: \times 60$; in $\mathbf{c}-\mathbf{f}: \times 225$; in $\mathbf{g}$ and $\mathbf{h}: \times 1,000$. In $\mathbf{i}$, bar $=2,000 \mathrm{~nm}$; in $\mathbf{j}$, bar $=5,000 \mathrm{~nm}$. all capillary loop-stage (immature) glomeruli, all of these endothelial cells lacked fenestrations. Upon glomerular maturation, no endothelial cells remained. The variability in this phenotype is likely due to the time of genomic excision of the VEGF-A floxed allele. VEGF starts to be expressed during the S-shape stage of glomerulogenesis, whereas nephrin-Cre-mediated excision takes place slightly later during the capillary loop stage (17). Thus it appears that transient expression of VEGF is sufficient to direct a reduced number of incoming endothelial cells but insufficient to maintain survival and proliferation of these cells. In addition, our results suggest that there is a threshold level required for VEGF to establish fenestrations that is not reached in VEGF-null glomeruli. VSMA, a marker of glomerular mesangial cells during glomerular development, was also absent from null glomeruli, demonstrating that mesangial cell differentiation and/or
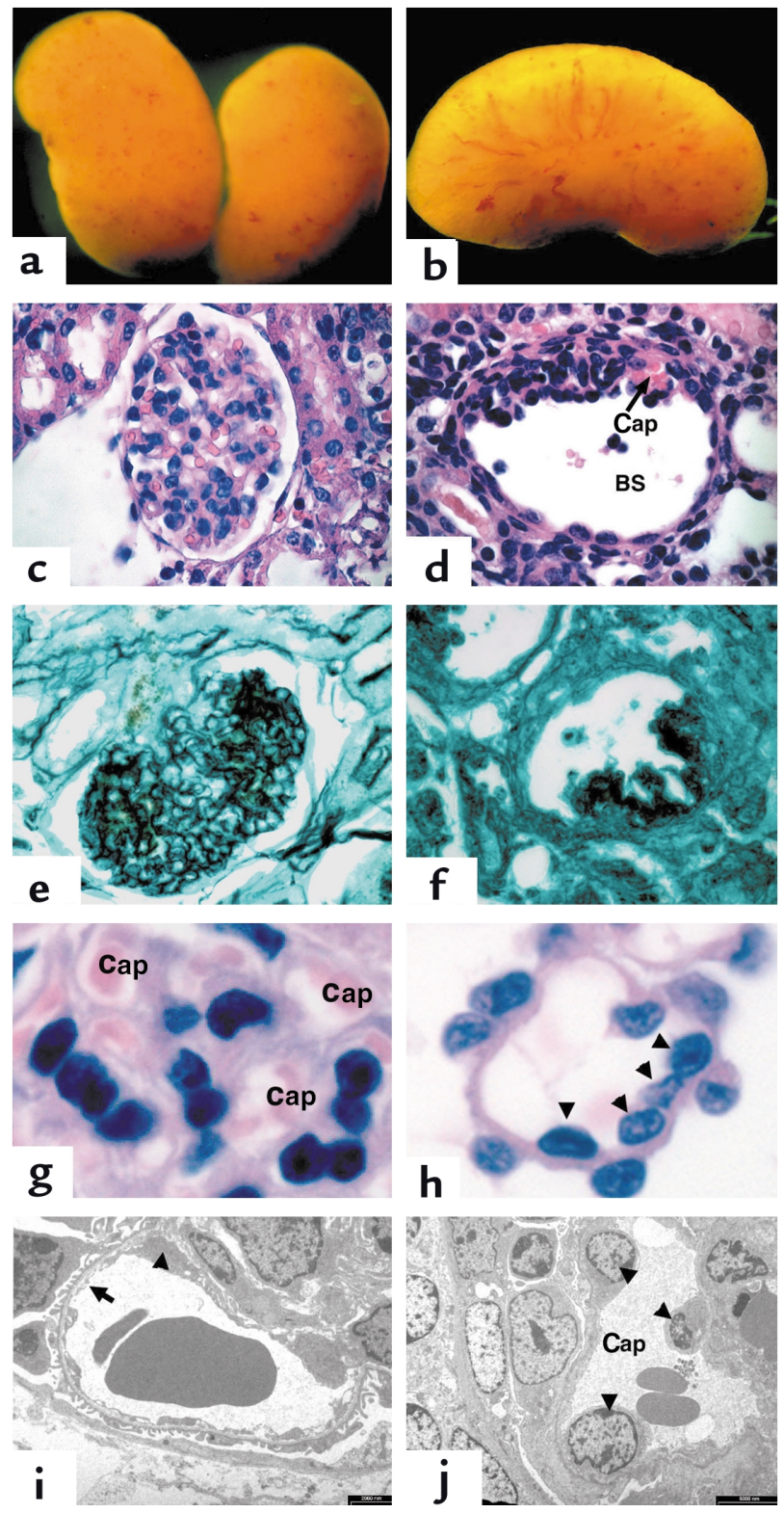
migration is dependent upon successful establishment of a glomerular capillary system.

In contrast, initial development of the glomerular filtration barrier was unaffected in the heterozygous VEGF-loxP $P^{+/-}, \mathrm{Neph}-\mathrm{Cre}^{+/-}$mice. However, by 2.5 weeks of age, marked swelling of the glomerular endothelial cells led to the appearance of bloodless glomeruli and endotheliosis, the pathognomonic lesion seen in preeclampsia. Although preeclampsia is a common and potentially fatal disease that affects $7-8 \%$ of all pregnancies, the pathogenesis of this disorder is poorly understood. Patients typically develop proteinuria, and renal biopsies performed early in the disease demonstrate endotheliosis that progresses to glomerulosclerosis in a subset of patients (21). By 9 weeks of age, all of the podocyte-specific VEGF heterozygotes developed end-stage kidney failure due to a severe form of glomerulosclerosis with loss of differentiated podocytes and endothelial cells. Although alterations in circulating levels of VEGF have been implicated in preeclampsia (25), the significance of these changes is unknown and has not been studied in tissues of affected organs such as the kidney. Our results suggest that downregulation of VEGF signaling within the glomerulus may be involved in the renal lesion of preeclampsia. Because the primary defect in preeclampsia is believed to lie in the placenta and/or trophoblast, it is interesting to speculate that some as-yet-unidentified factor that is generated by the placenta leads to downregulation of VEGF-A expression within the glomerulus and endotheliosis, and suggests future areas of potential investigation.

Although glomerular defects were not observed prior to 2.5 weeks of age, it is quite possible that earlier endothelial and/or podocyte defects exist that we were unable to detect. Furthermore, the dramatic loss of podocytes by 9 weeks of age suggests that upon stimulation with VEGF-A, endothelial cells "signal back" to the podocyte, and that endothelial cell damage disrupts these reciprocal signals, emphasizing the importance and dependence of reciprocal interactions between these two cell types.

In addition to its paracrine role in the glomerulus, it is possible that VEGF-A has an autocrine function that is required for podocyte survival. Presently, it is controversial whether Flk1 is expressed even at low levels in podocytes. However, we have crossed our nephrinCre recombinase mice with floxed Flk1 mice (a kind gift of J. Rossant's lab at The Samuel Lunenfeld Research Institute). By 4 weeks of age, these mice have no phenotype that demonstrates an absence of Flk1-dependent autocrine signaling within the podocyte. VEGF-A is required for breast cancer cell survival in vitro; in this setting, VEGF-A appears to signal in an autocrine fashion through the VEGF coreceptor, neuropilin-1, in the absence of Flk1 (26). As neuropilin-1 is expressed in the podocyte, additional studies that target neuropilin-1 in the podocyte are required to definitively answer this question.
Previous studies that have globally reduced the expression of VEGF-A in the mouse by using neutralizing antibodies $(16,27)$ or expressing only the 120 isoform of VEGF-A (28) have reported glomerular defects that are different from those seen in our study. The intraperitoneal injection of neutralizing antibodies to human recombinant VEGF in postnatal day 1-3 mice led to mesangiolysis and an arrest in postnatal kidney development. Similarly, the postnatal administration of a soluble chimeric VEGF receptor (Flt1) led to hypocellular glomeruli with mesangial deposits and mesangial cell vacuolization (16). In addition, the authors observed a decrease in the number of glomerular capillaries and fewer endothelial cell fenestrations (16). In the developing kidney, VEGF-A is expressed both in podocytes and in tubular epithelial cells and adjacent metanephric mesenchymal cells (29). The differences seen between previous studies and the present one are most likely due to alteration of VEGF levels in multiple cell populations within the kidney and to a variable reduction of the VEGF dose, which may be more difficult to control with a circulating antibody or receptor. In addition, endogenous VEGF-A levels were upregulated in podocytes in a study by Kitamoto et al. (27). In our model, there is a complete absence of VEGF in podocytes. The glomerular phenotype was more severe in our null mice than in mice treated with blocking antibodies or the soluble Flt receptor, suggesting that the localized delivery of VEGF from the podocyte across the heparan sulfate-rich GBM to the VEGF receptors that face the GBM (30) is critical for its function in vivo.

More recently, Carmeliet and colleagues have reported that mice that express only the secreted 120 isoform of VEGF-A develop glomerulosclerosis by 6 weeks of age (28). Although endothelial cells are lost, the podocytes are reportedly normal. In our experiments, all isoforms of VEGF-A are lost from the kidney and the phenotype differs from the VEGF-120 mice. Together, these results clearly emphasize the importance of isoform-specific functions of VEGF-A within the glomerulus. Genomic targeting experiments that will address the role of the different VEGF isoforms within the podocyte are underway.

Given the exquisite sensitivity to VEGF dosage reduction in glomerular development and function, we also sought to determine the phenotype resulting from overexpression of the 164 isoform of VEGF-A specifically in the podocyte. The 164 isoform is secreted and bound by heparan sulfate in the GBM. These transgenic mice developed a dramatic glomerular phenotype and rapidly succumbed to end-stage renal failure. At the time of death, the majority of their glomeruli demonstrated global collapse of the tuft as seen in collapsing glomerulopathy and HIVAN (14). Why do the capillaries collapse? At birth and from 1-5 days of age, the glomeruli are present and filter urine. At this time, the patent glomerular capillary loops have greater diameters and a greater number of 
endothelial cell nuclei than do wild-type capillaries. Other studies have shown that treatment of endothelial cells with increasing doses of VEGF-A leads to coalescence of endothelial cells and the formation of larger endothelial tubes, a process that has been termed "hyperfusion" $(31,32)$. In the absence of increased glomerular capillary flow, this would lead to a fall in intraluminal capillary pressure and collapse. Of clinical relevance, the tat protein from HIV has been shown to signal through Flk1 in endothelial cells in Kaposi sarcoma (33-35), and the podocyte has been identified as a reservoir for the HIV virus (36). Taken together, these results present a possible explanation for the similarity between the capillary collapse seen in the VEGF-A overexpression model and HIVAN.

It has been hypothesized that damage to the podocyte ultimately leads to the capillary collapse seen in HIVAN and other forms of collapsing glomerulopathies (37-40). However, our results demonstrate that capillary collapse can occur in the absence of dedifferentiation or dysregulation of podocytes. In fact, the capillaries also collapse in heterozygous VEGF-loxP $\mathrm{P}^{+-}, \mathrm{Neph}-\mathrm{Cre}^{+/-}$mice after the endothelial cells are lost, and in this case, the differentiated podocytes are lost. Thus, it is evident that a single mechanism or phenotype cannot explain all cases of capillary collapse in glomerular disease.

In summary, our results demonstrate an exquisite dosage sensitivity for VEGF-A in the developing glomerulus. Numerous clinical studies have documented that alterations in glomerular VEGF-A expression are associated with glomerular disease (41-44). Our results demonstrate that dysregulation of VEGFA is not only associated with but also plays a pathogenic role in initiating glomerular injury. The Cre-loxP system and transgenic approach allowed us to engineer mice with three different doses of VEGF within the podocyte based on the allele copy number. Each VEGF level was associated with a distinct mechanism that led to one of three important glomerular phenotypes. These results provide insight into the molecular mechanisms that underlie a variety of common and clinically important human diseases, including preeclampsia and HIV, and suggest potential future avenues for therapeutic intervention. In addition, it is clear that interactions between podocytes and endothelial cells are critical during development of the glomerular filtration barrier and continue in the filtering glomerulus.

Finally, these results provide a note of caution for clinical trials aimed at altering VEGF levels. Although the podocyte has not been specifically targeted in these therapies, careful monitoring of renal function with a particular emphasis on the glomerular filtration barrier should be included in the clinical protocols.

\section{Acknowledgments}

We gratefully acknowledge the technical support of Lois Schwartz for generation of transgenic mice, Doug Holmyard for EM processing and images, the Toronto
Centre for Comparative Models of Human Disease for help with biochemical analysis of the mice, and Dragana Vukasovic for excellent secretarial assistance. We also thank Janet Rossant and Jordan Kreidberg for critically reviewing the manuscript and Wilhelm Kriz for invaluable assistance. S.E. Quaggin is the recipient of a Canada Research Chair. This work was funded by NIH grant 5 R21 DK-59148-02 and a Kidney Foundation of Canada Grant (to S.E. Quaggin). This work was inspired by the courage of Kelly Kalt (1983-2000).

1. Mundel, P., and Reiser, J. 1997. New aspects of podocyte cell biology. Kidney Blood Press. Res. 20:173-176.

2. Abrahamson, D.R. 1991. Glomerulogenesis in the developing kidney. Semin. Nephrol. 11:375-389.

3. Kriz, W., and Lemley, K. 1999. The role of the podocyte in glomerulosclerosis. Curr. Opin. Nephrol. Hypertens. 8:489-497.

4. Kriz, W. 1997. Evolving role of the podocyte in chronic renal failure. Kidney Blood Press. Res. 20:180-183.

5. Shirato, I., et al. 1996. The development of focal segmental glomerulosclerosis in masugi nephritis is based on progressive podocyte damage. Virchows Arch. 429:255-273.

6. Coimbra, T.M., et al. 2000. Early events leading to renal injury in obese Zucker (fatty) rats with type II diabetes. Kidney Int. 57:167-182.

7. Floege, J., et al. 1997. Age-related glomerulosclerosis and interstitial fibrosis in Milan normotensive rats: a podocyte disease. Kidney Int. 51:230-243.

8. Pagtalunan, M.E., et al. 1997. Podocyte loss and progressive glomerular injury in type II diabetes. J. Clin. Invest. 99:342-348.

9. Robert, B., Zhao, X., and Abrahamson, D.R. 2000. Coexpression of neuropilin-1, flk1, and VEGF(164) in developing and mature mouse kidney glomeruli. Am. J. Physiol. Renal Physiol. 279:F275-F282.

10. Saxen, L., and Sariola, H. 1987. Organogenesis of the kidney. Pediatr. Nephrol. 1:385-392.

11. Carmeliet, P., et al. 1996. Abnormal blood vessel development and lethality in embryos lacking a single VEGF allele. Nature. 380:435-439.

12. Ferrara, N., et al. 1996. Heterozygous embryonic lethality induced by targeted inactivation of the VEGF gene. Nature. 380:439-442.

13. Somlo, S., and Mundel, P. 2000. Getting a foothold in nephrotic syndrome. Nat. Genet. 24:333-335.

14. Laurinavicius, A., Hurwitz, S., and Rennke, H.G. 1999. Collapsing glomerulopathy in HIV and non-HIV patients: a clinicopathological and follow-up study. Kidney Int. 56:2203-2213.

15. Novak, A., Guo, C., Yang, W., Nagy, A., and Lobe, C.G. 2000. Z/EG, a double reporter mouse line that expresses enhanced green fluorescent protein upon Cre-mediated excision. Genesis. 28:147-155.

16. Gerber, H.P., et al. 1999. VEGF is required for growth and survival in neonatal mice. Development. 126:1149-1159.

17. Eremina, V., Wong, M.A., Cui, S., Schwartz, L., and Quaggin, S.E. 2002. Glomerular-specific gene excision in vivo. J. Am. Soc. Nephrol. 13:788-793.

18. Laemmli, U.K. 1970. Cleavage of structural proteins during the assembly of the head of bacteriophage T4. Nature. 227:680-685.

19. Wong, M.A., Cui, S., and Quaggin, S.E. 2000. Identification and characterization of a glomerular-specific promoter from the human nephrin gene. Am. J. Physiol. Renal Physiol. 279:F1027-F1032.

20. Miner, J.H., and Li, C. 2000. Defective glomerulogenesis in the absence of laminin alpha5 demonstrates a developmental role for the kidney glomerular basement membrane. Dev. Biol. 217:278-289.

21. Kincaid-Smith, P. 1991. The renal lesion of preeclampsia revisited. Am. J. Kidney Dis. 17:144-148.

22. McDonald, R., Wiggelinkhuizen, J., and Kaschula, R.O. 1971. The nephrotic syndrome in very young infants. Am. J. Dis. Child. 122:507-512.

23. Esser, S., et al. 1998. Vascular endothelial growth factor induces endothelial fenestrations in vitro. J. Cell Biol. 140:947-959.

24. Roberts, W.G., and Palade, G.E. 1995. Increased microvascular permeability and endothelial fenestration induced by vascular endothelial growth factor. J. Cell Sci. 108:2369-2379.

25. Bielecki, D.A., Klonowska-Dziatkiewicz, E., Jarocki, S., and Urban, J. 2002. Growth factors in pregnancy complications with preeclampsia. Ginekol Pol. 73:422-429.

26. Bachelder, R.E., et al. 2001. Vascular endothelial growth factor is an autocrine survival factor for neuropilin-expressing breast carcinoma cells. Cancer Res. 61:5736-5740.

27. Kitamoto, Y., Tokunaga, H., and Tomita, K. 1997. Vascular endothelial growth factor is an essential molecule for mouse kidney development: glomerulogenesis and nephrogenesis. J. Clin. Invest. 99:2351-2357. 
28. Mattot, V., et al. 2002. Loss of the VEGF(164) and VEGF(188) isoforms impairs postnatal glomerular angiogenesis and renal arteriogenesis in mice. J. Am. Soc. Nephrol. 13:1548-1560.

29. Miquerol, L., Gertsenstein, M., Harpal, K., Rossant, J., and Nagy, A. 1999. Multiple developmental roles of VEGF suggested by a LacZtagged allele. Dev. Biol. 212:307-322.

30. Gengrinovitch, S., et al. 1999. Glypican-1 is a VEGF165 binding proteoglycan that acts as an extracellular chaperone for VEGF165. J. Biol. Chem. 274:10816-10822.

31. Drake, C.J., and Little, C.D. 1995. Exogenous vascular endothelial growth factor induces malformed and hyperfused vessels during embryonic neovascularization. Proc. Natl. Acad. Sci. U. S. A. 92:7657-7661.

32. Drake, C.J., and Little, C.D. 1999. VEGF and vascular fusion: implications for normal and pathological vessels. J. Histochem. Cytochem. 47:1351-1356.

33. Morini, M., et al. 2000. Kaposi's sarcoma cells of different etiologic origins respond to HIV-Tat through the Flk-1/KDR (VEGFR-2): relevance in AIDS-KS pathology. Biochem. Biophys. Res. Commun. 273:267-271.

34. Ganju, R.K., et al. 1998. Human immunodeficiency virus tat modulates the Flk-1/KDR receptor, mitogen-activated protein kinases, and components of focal adhesion in Kaposi's sarcoma cells. J. Virol. 72:6131-6137.

35. Vene, R., Benelli, R., Noonan, D.M., and Albini, A. 2000. HIV-Tat dependent chemotaxis and invasion, key aspects of tat mediated pathogenesis. Clin. Exp. Metastasis. 18:533-538.
36. Marras, D., et al. 2002. Replication and compartmentalization of HIV-1 in kidney epithelium of patients with HIV-associated nephropathy. Nat. Med. 8:522-526.

37. Barisoni, L., Kriz, W., Mundel, P., and D'Agati, V. 1999. The dysregulated podocyte phenotype: a novel concept in the pathogenesis of collapsing idiopathic focal segmental glomerulosclerosis and HIV-associated nephropathy. J. Am. Soc. Nephrol. 10:51-61.

38. Bruggeman, L.A., et al. 1997. Nephropathy in human immunodeficiency virus-1 transgenic mice is due to renal transgene expression. J. Clin. Invest. 100:84-92.

39. Conaldi, P.G., et al. 2002. Human immunodeficiency virus-1 tat induces hyperproliferation and dysregulation of renal glomerular epithelial cells. Am. J. Pathol. 161:53-61.

40. Husain, M., et al. 2002. HIV-1 Nef induces proliferation and anchorageindependent growth in podocytes. J. Am. Soc. Nephrol. 13:1806-1815.

41. Shahbazi, M., et al. 2002. Vascular endothelial growth factor gene polymorphisms are associated with acute renal allograft rejection. J. Am. Soc. Nephrol. 13:260-264.

42. Kim, Y.G., et al. 2000. Vascular endothelial growth factor accelerates renal recovery in experimental thrombotic microangiopathy. Kidney Int. 58:2390-2399.

43. Cha, D.R., et al. 2000. Role of vascular endothelial growth factor in diabetic nephropathy. Kidney Int. Suppl. 77:S104-S112.

44. Ostendorf, T., et al. 1999. VEGF(165) mediates glomerular endothelial repair. J. Clin. Invest. 104:913-923. 